\title{
X-ray Tomography Generates Quantitative, 3-D Views of Intact Cells
}

\author{
M. Uchida*, C. Knoechel*, G. McDermott*, M. Wetzler**, A. Barron**, M.A. Le Gros, ${ }^{* * *}$ and \\ C.A. Larabell***** \\ * Department of Anatomy, University of California, San Francisco, CA 94143-2722 ** \\ Bioengineering Department, Stanford University, Palo Alto, CA $94301 * * *$ Physical Biosciences \\ Division, Lawrence Berkeley Laboratory, Berkeley CA 94720
}

Soft X-ray tomography (SXT) combines features associated with light and electron microscopy it is an imaging technique that is both fast and relatively easy to accomplish (like light microscopy) that produces high-resolution, absorption-based images (like electron microscopy). With SXT, we can examine whole, hydrated cells (between 10-15 $\mu \mathrm{m}$ thick), eliminating the need for time-consuming and potentially artifact-inducing embedding and sectioning procedures. SXT is similar in concept to the well-established medical diagnostic technique, computed axial tomography (CAT), except SXT is capable of imaging with a spatial resolution of $50 \mathrm{~nm}$, or better. In SXT, cells are imaged using X-ray energies between the $\mathrm{K}$ shell absorption edges of carbon $(284 \mathrm{eV}, \lambda=4.4 \mathrm{~nm})$ and oxygen $(543 \mathrm{eV}, \lambda=2.3 \mathrm{~nm})$. In this energy range, photons readily penetrate the aqueous environment while encountering significant absorption from carbon- and nitrogen-containing organic material. Consequently organic material absorbs approximately an order of magnitude more strongly than water, producing a quantifiable natural contrast image of cellular structures. SXT, like other tomography modalities, requires recording images from multiple different viewing angles. By collecting images from multiple angles through 180 degrees of rotation, SXT reconstructions yield information at isotropic resolution. The positions of molecules can be determined using heavy metal tags, such as gold and titanium dioxide, or by overlaying images from correlated cryo fluorescence and X-ray imaging ${ }^{2}$.

Images are formed using unique optics called zone plates (ZP). An X-ray ZP optic consists of a number of concentric nanostructured metal rings, or zones, formed on a thin X-ray transmissive silicon nitride membrane. The width of the outermost ring determines the spatial resolution of the $\mathrm{ZP}$ lens, whereas the thickness of the rings determines the focusing efficiency. In the microscope we utilize, the condenser ZP lens has an overall diameter of $1 \mathrm{~cm}$ and an outer zone width of $50 \mathrm{~nm}$. The high-resolution objective ZP lens has a diameter of $63 \mu \mathrm{m}, 618$ zones, a focal length of $650 \mu \mathrm{m}$ at $2.4 \mathrm{~nm}$ wavelength and an outer zone width of $25 \mathrm{~nm}$. This produces a numerical aperture of 0.05 . The resolution of the instrument is most dependent on the quality and diameter of the objective ZP lens, which is limited by the accuracy of current nanometer fabrication technology. The best ZP available to date is one that generates $15 \mathrm{~nm}$ resolution ${ }^{4}$.

Because SXT is fast ( $~ 5$ min per tomographic data set), we can examine large numbers of cells. We demonstrated this capability by using SXT to evaluate the effect of peptoids on the pathogenic yeast, Candida albicans. We examined the subcellular changes that occur as a consequence of both phenotypic switching and of treating $\mathrm{C}$. albicans with antifungal peptoids, a class of candidate therapeutics unaffected by drug resistance mechanisms ${ }^{3}$. Peptoid treatment suppressed formation of the pathogenic hyphal phenotype and resulted in striking changes in cell and organelle morphology, most dramatically in the nucleus and nucleolus, and in the number, size, and location of lipidic bodies. In particular, peptoid treatment was seen to cause the inclusion of lipidic bodies into the nucleus ${ }^{3}$. 


\section{References}

[1] M.A. Le Gros, et. al. Current Opinion in Structural Biology 15 (2005) 593.

[2] M.A. Le Gros, et. al. J. Microscopy 235 (2009) 1.

[3] M. Uchida et. al. PNAS 106 (2009) 19375.

This work was funded by the Department of Energy Office of Biological and Environmental Research Grant DE-AC02- 05CH11231, the National Institutes of Health (NIH) National Institute for Allergy and Infectious Diseases Grant GM072666, and the NIH National Center for Research Resources Grant RR019664. 